Donkervoort M, Dekker J, Deelman B.

The course of apraxia and ADL functioning in left hemisphere stroke patients treated in rehabilitation centres and nursing homes.

Clinical Rehabilitation: 20, 2006, nr. 12, p. 1085-1893

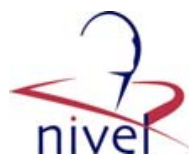

\begin{tabular}{|l|l|}
\hline Postprint Version & 1.0 \\
\hline Journal website & $\underline{\text { http://www.sagepub.co.uk/journalsProdDesc.nav?prodId=Journal201806 }}$ \\
\hline Pubmed link & $\underline{\text { http://www.ncbi.nlm.nih.gov/entrez/query.fcgi?db=pubmed\&cmd=Retrieve\&dop }}$ \\
\hline DO=AbstractPlus\&list_uids=17148520\&query_hl=4\&itool=pubmed_docsum
\end{tabular}

\title{
The course of apraxia and ADL functioning in left hemisphere stroke patients treated in rehabilitation centres and nursing homes
}

\author{
MireILLE DONKERVOORT ${ }^{1 *}$, JOOST DEKKER ${ }^{2}$ AND BETTO DEELMAN $^{3}$ \\ ${ }^{1}$ Netherlands Institute of Health Services Research (Nivel), Utrecht and Department of Rehabilitation \\ Medicine, Erasmus Medical Centre, Rotterdam \\ ${ }^{2}$ Department of Rehabilitation Medicine, VU University Medical Centre, Amsterdam \\ ${ }^{3}$ University of Groningen, Groningen, The Netherlands \\ *Address for correspondence: M Donkervoort, Erasmus MC, Dept. Rehabilitation Medicine, PO Box \\ 2040, 3000 CA Rotterdam, The Netherlands. E-mail: M.Donkervoort@ErasmusMC.nl
}

Objective: To study the course of apraxia and daily life functioning (ADL) in left hemisphere stroke patients with apraxia.

Design: Prospective cohort study.

Setting: Rehabilitation centres and nursing homes.

Subjects: One hundred and eight left hemisphere stroke patients with apraxia, hospitalized in rehabilitation centres and nursing homes.

Measures: ADL-observations, Barthel ADL Index, Apraxia Test, Motricity Index.

Results: During the study period of 20 weeks, patients showed small improvements in apraxia (standardized mean differences of 0.19 and 0.33) and medium-sized improvements in ADL functioning (standardized mean differences from 0.37 to 0.61 ). About $88 \%$ of the patients were still apraxic at week 20. Less improvement in apraxia was observed in initially less severe apraxic patients. Less improvement in ADL functioning was found to be associated with more severe apraxia, a more independent initial ADL score, higher age, impaired motor functioning and longer time between stroke and first assessment.

Conclusions: Apraxia in stroke patients is a persistent disorder, which has an adverse influence on ADL recovery.

\section{INTRODUCTION}

Apraxia is the inability to carry out learned and purposeful activities; it is an inability that is not explained by primary motor or sensory impairments, or deficits in motivation, memory or comprehension. ${ }^{1-3}$ Apraxia is a common cognitive impairment after a left hemisphere stroke. It is estimated that approximately $30 \%$ of patients with left hemisphere stroke have apraxic

impairments. ${ }^{1,45}$ Although the incidence of apraxia is high, it is frequently believed that the condition recovers spontaneously. ${ }^{6-8}$ Therefore, little attention is paid to the treatment and management of apraxia. Some studies, however, have observed the disorder to be enduring. ${ }^{6,9}$ This raises the question what the course of apraxia is and which factors influence its course. Similarly, some have questioned 


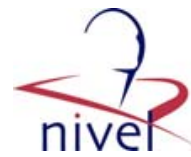

whether apraxia has lasting effects on ADL functioning, while others have suggested apraxia to have long-term, real-life consequences. ${ }^{10-15}$ The few studies on this issue showed contrasting results. ${ }^{10-15}$

Thus, the aim of the present study is: (1) to study the course of apraxia in stroke patients; (2) to identify factors that affect the course of apraxia; (3) to study the course of ADL functioning in stroke patients with apraxia; and (4) to determine whether the severity of apraxia and other factors affect the course of ADL functioning.

\section{METHODS}

The data used in this study were collected as part of a randomized clinical trial comparing strategy training to usual treatment in left hemisphere stroke patients with apraxia. ${ }^{16}$ Patients from both treatment groups (strategy training or usual treatment) participated: type of treatment was controlled for in all regression analyses (see Statistical analyses). Forty-nine institutions (15 rehabilitation centres and 34 nursing homes) in the Netherlands participated in the study. The patient inclusion criteria were: (1) left hemisphere stroke, (2) apraxia and (3) staying on an inpatient care unit. Exclusion criteria were: (1) history of apraxia before current stroke; (2) stroke had occurred less than four weeks or more than two years ago; (3) age younger than 25 years or older than 95 years; (4) a history of brain damage in combination with a period of coma lasting longer than $15 \mathrm{~min}$ or a period of post-traumatic amnesia existing longer than 2 hours; (5) history of brain tumour; (6) no working knowledge of the Dutch language; (7) premorbid or present pathologies such as a psychiatric or psycho-geriatric history, addiction to alcohol, medical or other drugs; (8) premorbid personality, intellectual or learning disorders; (9) a history of serious consciousness impairments; and (10) the treating physician, the occupational therapist, and/or the patient did not judge the treatment of apraxia to be necessary.

A left hemisphere stroke was diagnosed when acute clinical symptoms of a focal dysfunction of the left hemisphere were present; when these signs and symptoms lasted at least 24 hours, and when there was no other than a vascular origin. ${ }^{17}$ The diagnosis of apraxia consisted of two steps. First, a clinical diagnosis of apraxia was made by the patients' treating medical team. The patient was diagnosed as apraxic if (a) the patient showed the inability (or restriction in the ability) to carry out purposeful activities and (b) this inability was not the result of a primary motor or sensory impairment, or deficit of comprehension or motivation..$^{1-3}$ Second, patients were tested for apraxia with a recently developed neuropsychological apraxia test ${ }^{5,18}$ by a trained researcher. Patients who showed no apraxia on this test (score above 87; see Measurements section) were excluded from the study.

The patients (and their families) received verbal and written information about the study and gave verbal as well as written consent to participate. From 139 patients selected, 26 patients were eventually not included in the randomized clinical trial because they scored above 87 on the apraxia test $(n=13)$, withdrew their consent $(n=5)$, or were discharged from the institute $(n=8)$. In addition, five patients dropped out of the study just before the first assessments (withdrew consent $(n=3)$, discharged $(n=1)$, deceased $(n=1))$. In total 108 patients with a left hemisphere stroke diagnosed as apraxic participated in the present study.

The patients stayed on an inpatient care unit in a rehabilitation centre or nursing home where they were treated by a medical team. Because of the randomized controlled trial $50 \%$ of the patients received strategy training integrated in the usual occupational therapy, the other $50 \%$ received the usual occupational therapy only. ${ }^{16}$ In the present study the analyses were controlled for type of treatment.

\section{Measurements}

The data in the present study were collected during three assessments: (1) at baseline, (2) post treatment (i.e. eight weeks after baseline) and (3) at follow-up (i.e. 20 weeks after baseline). Patients were assessed on apraxia, motor functioning and daily life functioning (ADL) by a trained research assistant. Furthermore, at baseline demographic and clinical data were collected.

\section{Demographic and clinical data}

The following information was recorded: gender, age, handedness (right/left), type of stroke (haemorrhage/ infarction and first/recurrent), hemiplegia/ hemiparesis (yes/no), disease duration, type 
Donkervoort M, Dekker J, Deelman B.

The course of apraxia and ADL functioning in left hemisphere stroke patients treated in

rehabilitation centres and nursing homes.

Clinical Rehabilitation: 20, 2006, nr. 12, p. 1085-1893

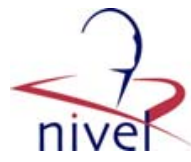

of institution (rehabilitation centre/nursing home) and trial treatment allocation (strategy training/ usual therapy).

\section{Apraxia}

The Apraxia Test measures the severity of apraxia. ${ }^{5,18}$ The test is based on the work of De Renzi ${ }^{1}$ and consists of 15 items assessing the ability to use or mime objects and the ability to imitate gestures. Patients are asked to use their unaffected ipsilateral arm. The maximum score per item is 6 , the maximum score of the total test is 90 . The internal consistency, validity and inter-rater reliability of the test is good. ${ }^{5,18}$

\section{Motor functioning}

The Motricity Index ${ }^{19}$ was used to assess motor impairments of the affected contralateral arm and leg. In addition to the Motricity Index the patients sitting balance was measured (Trunk Control Test ${ }^{20}$ ). If the patient was able to maintain sitting balance during minimal 30 seconds without support, sitting balance was scored 1 , otherwise sitting balance was scored 0 . The tests are well standardized, with proven reproducibility, validity and sensitivity to change. ${ }^{21,22}$

\section{ADL functioning}

Two measures to assess ADL functioning were used: ADL-observations and Barthel ADL Index. The ADL-observations ${ }^{23,24}$ is a set of standardized observations specially developed to assess disabilities due to apraxia. Four daily activities (personal hygiene, dressing, prepare sandwich, prepare hot chocolate) were observed and scored with respect to independence and performance (deficits in initiation, execution and control of the activity). In this study the overall mean score of these observations was used, ranging from 0 (dependent) to 3 (independent). The internal consistency and interobserver reliability of this observation procedure are found to be good. ${ }^{23,24}$

The Barthel ADL Index ${ }^{25,26}$ is a widely used and standard measure of ADL functioning. The patient's dependency is scored on 10 basic daily functions/activities (bowel control, bladder control, grooming, toilet use, feeding, transfer, mobility, dressing, using the stairs, bathing). The Barthel ADL Index expresses disability on a scale ranging from 0 (totally dependent) to a maximum score of 20 (totally independent).

\section{Statistical analysis}

The dependent variables in this study comprise change scores on the apraxia test, the standardized ADL-observations and the Barthel ADL Index: the changes from baseline to week 8 and from baseline to week 20 were calculated. The changes in apraxia and ADL functioning were tested for significance with a paired-sample $t$-test $(P<0.01)$ and was expressed by standardized mean differences (change score divided by the standard deviation of the baseline score). A standardized mean difference of 0.2 was regarded as small, of 0.5 as medium, and 0.8 as large. ${ }^{27}$ All variables measured at baseline were used as independent variables. Pearson's correlation coefficients were computed to examine possible relations between the independent and dependent variables. Multiple regression analyses were performed using the independent variables that showed a significant $(P \leq 0.05)$ univariate relationship with at least one of the three dependent variables. All regression analyses were controlled for type of treatment (strategy training or usual treatment). The independent variables were entered stepwise in the multiple regression analyses. Probability of $F$ to enter the analysis was set at $P \leq 0.05$, and the probability to remove was set at $P \geq 0.1$. The standardized regression coefficients (beta) and final explained variance $\left(R^{2}\right)$ are presented.

\section{RESULTS}

The demographic and clinical characteristics of the 108 participating patients are presented in Table 1. Although $90 \%$ of the patients were able to sit independently for at least 30 seconds, they also experienced considerable motor impairments. According to the Motricity Index approximately $35 \%$ of the patients were not able to show any movement in the hemiplegic side of their body, while only $5 \%$ of the patients had normal strength in the right side of their body. Furthermore, the patients showed considerable disabilities on the measurements of ADL functioning. About $44 \%$ of the patients could 
be classified as (very) severely disabled (score below 10 on the Barthel ADL Index). ${ }^{28,29}$ The mean score on the ADL-observations at baseline was 2.3 (maximum score is 3). Approximately $90 \%$ of the patients scored worse than the mean score of 2.8 found in a group of left hemisphere stroke patients without apraxia. $^{24}$

\section{[ TABLE 1 ]}

\section{Course of apraxia}

Figure 1 shows the course of apraxia. Patients had improved on average 3.7 points (SD 12.4; $P<0.01$ ) on the apraxia test at week 8. At week 20 the patients had improved 6.3 points (SD 14.6; $P<0.01$ ). The standardized mean differences representing the improvement at week 8 and week 20 were small, respectively 0.19 and 0.33 . After eight weeks $10 \%$ of the patients $(n=11)$ obtained a score above 87 (indicating no apraxia) and after 20 weeks this percentage was $12 \%(n=14)$. None of the participating patients scored above 87 at baseline (see exclusion criteria).

\section{[ FIGURE 1 ]}

\section{Prediction of the course of apraxia}

The univariate analyses (Table 2) show that less improvement in apraxia at week 8 is significantly correlated with less severe apraxia at baseline and a longer time period between stroke and the baseline assessment. At week 20, less improvement in apraxia is correlated with less severe apraxia at baseline and gender (e.g. women showed less improvement at week 20).

\section{[ TABLE 2 ]}

In the multiple regression analyses, all variables that showed significant univariate correlations with one of the change scores (see Table 2) were used in the regression analyses, except type of institution because of its collinearity with age. The obtained regression models (Table 3 ) for change in apraxia at week 8 and week 20 explained $22 \%$ and $27 \%$ of the total variance, respectively. Patients with less severe apraxia at baseline showed less improvement on the apraxia test at week 8 and week 20 , compared to patients with more severe signs of apraxia at baseline. A longer time period between stroke and baseline assessments was associated with less improvement in apraxia at week 8 . This association was not found at week 20. Furthermore, women showed somewhat less improvement than men at week 20.

\section{[ TABLE 3 ]}

\section{Course of ADL functioning}

Figure 2 shows the recovery patterns in ADL functioning. On the ADL-observations, the patients had improved on average 0.18 points (SD $0.35 ; P<0.01$ ) at week 8 . At week 20 , the patients had improved 0.23 points (SD $0.41 ; P<0.01$ ). The standardized mean differences representing the improvement at week 8 and week 20 are of medium size, respectively 0.37 and 0.48 . The percentage of patients that scored maximum on the ADL-observations raised from $5 \%(n=5)$ at baseline to $13 \%(n=11)$ at week 8 and $24 \%(n=18)$ at week 20 .

\section{[ FIGURE 2 ]}

On the Barthel ADL Index the mean improvement was 2 points (SD 2.8; $P<0.01$ ) at week 8. At week 20 the patients had improved 3 points (SD 3.4; $P<0.01$ ). The matching standardized mean differences are of medium size, respectively 0.41 and 0.61 . The percentage of patients that obtained a maximum 
Donkervoort M, Dekker J, Deelman B.

The course of apraxia and ADL functioning in left hemisphere stroke patients treated in

rehabilitation centres and nursing homes.

Clinical Rehabilitation: 20, 2006, nr. 12, p. 1085-1893

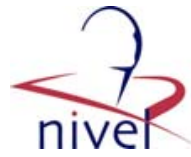

score on the Barthel ADL Index raised from 7\% $(n=7)$ at baseline to $11 \%(n=10)$ at week 8 and 20. The percentage of patients classified as (very) severely disabled decreased from $44 \%(n=41)$ on the first assessment to respectively $23 \%(n=21)$ and $16 \%(n=12)$ at week 8 and 20.

\section{Prediction of the course of ADL functioning}

The univariate analyses (Table 2) show that less improvement on the ADL-observations at week 8 and week 20 is correlated with a more independent ADL-observation score at baseline. In addition, urinary continence is correlated with less improvement on the ADL-observations at week 20.

In the multiple regression analyses all variables that showed a significant univariate correlation with one of the change scores were used (see Table 2), except type of institution because of its collinearity with age. The obtained multiple regression models (Table 3 ) for change on the ADL-observations at week 8 and week 20 explained $25 \%$ and $41 \%$ of the total variance, respectively. A more independent ADL-observation score at baseline and higher age were found to be correlated with less improvement on the ADL-observations at week 8 and week 20. More severe apraxia at baseline resulted in less improvement on the ADL-observations at week 8. In addition it was shown that urinary continent patients and patients with a lower (less independent) initial score on the Barthel had improved less on the ADL-observations at week 20.

The univariate analyses (Table 2) show significant correlations between change on the Barthel ADL Index and initial Barthel score, time since stroke, urinary continence, age and type of institution. In the multiple regression analyses the same variables were used as in the regression analyses of the change on the ADL-observations. The obtained regression models for improvement on the Barthel ADL Index at week 8 and week 20 explained $36 \%$ and $35 \%$ of the total variance, respectively. A more independent initial score on the Barthel ADL Index, higher age and a larger time interval between stroke and the first assessment were associated with less improvement on the Barthel ADL Index at week 8 and week 20. In addition, impaired motor functioning was associated with less improvement on the Barthel ADL Index at week 8.

\section{DISCUSSION}

Although it has been believed that apraxia recovers spontaneously, ${ }^{7}$ the results of the present study state otherwise and thereby support the few studies that show apraxia to be persistent. ${ }^{6,9}$ Over a period of five months our apraxic patient group showed a small improvement on the apraxia test (standardized mean difference of 0.33 ). At week 20 , only about $10 \%$ of the studied patients showed no longer signs of apraxia on the apraxia test. The initially less severe apraxia patients showed less improvement in apraxia (week 8 and 20). The analyses did not show other consistent predictors of apraxia recovery.

With respect to ADL functioning, improvement was more profound. The standardized mean differences matching the improvement in ADL functioning were of medium size, ranging from 0.37 to 0.61. The most important factor found to be associated with improvement in ADL functioning was again - the initial score on the outcome measure. A higher initial score (e.g. less problems in ADL functioning) was associated with less improvement in ADL functioning. Because of possible ceiling effects additional analyses were performed, leaving out all patients who had a high initial score on the measures for ADL functioning (approximately the top 15\%). These additional analyses yielded similar correlations (results not shown). We found that in the first eight weeks, apraxia had a negative influence on ADL improvement measured by the ADL-observations, whereas motor impairments had a negative influence on the Barthel ADL Index. These results are consistent with our previous finding that the ADL-observations are more sensitive to apraxia, whereas the Barthel ADL Index is more sensitive to motor impairments. ${ }^{24,30}$ At week 20 , apraxia and motricity were not found to be associated with ADL functioning. This shows that long-term negative influences of apraxia and motor impairments on improvement in ADL functioning are not confirmed. It is possible that, with time, patients have learned to compensate for their impairments. All ADL functioning regression models showed that higher age was associated with less improvement in ADL functioning. Another consistent finding was the negative influence of a larger time interval (between stroke and the first assessment), on improvement in ADL functioning as measured by the Barthel ADL index. 
Donkervoort M, Dekker J, Deelman B.

The course of apraxia and ADL functioning in left hemisphere stroke patients treated in rehabilitation centres and nursing homes.

Clinical Rehabilitation: 20, 2006, nr. 12, p. 1085-1893

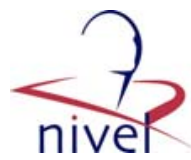

The present study supports findings from previous studies ${ }^{31}$ that initial ADL score, age and severity of paralysis (motor functioning) are important predictors for functional recovery of stroke patients. The earlier findings that a previous stroke, urinary incontinence and impaired sitting balance are negatively associated with functional recovery were not supported in our study.

Two important limitations in this study were caused by the fact that we used data from our randomized controlled trial on the effect of strategy training in stroke patients with apraxia. ${ }^{16}$ The selection criteria caused homogeneity in our patient group which was important for the randomized trial; but it is not clear whether the results can be generalized to patients not fitting the selection criteria. Furthermore, all patients received treatment from an occupational therapist regarding their problems in ADL functioning. Thus, we cannot draw conclusions concerning the course of apraxia and ADL functioning in nontreated patients. We expect non-treated patients to show less improvement in apraxia and ADL functioning.

In conclusion, the present study shows that apraxia is persistent - for at least six months - in a considerable number of stroke patients. There were no major predictors found for recovery of apraxia, except for the initial apraxia score: the initially more severe apraxic patients showed more improvement in apraxia. The patients showed improvement in ADL functioning. Less improvement in ADL functioning was consistently found to be associated with a more independent initial ADL score, higher age, and a longer time between stroke and first assessment. In the short term (week 8) a negative influence of apraxia and motor impairments on improvement in ADL functioning was observed.

\section{CLINICAL MESSAGES}

- Clinicians should be alert that apraxia may not recover spontaneously.

- Recently acquired apraxia has a negative influence on ADL functioning.

\section{ACKNOWLEDGEMENTS}

We thank all the participating institutes, and we are especially grateful to the patients and occupational therapists who participated. We also thank J Panis and other research assistants for conducting the measurements. Furthermore, we would like to thank the Netherlands Heart Foundation (grant number 95.009) and the Health Insurance Funds Council for their financial support.

\section{TABLES AND FIGURES}

Table 1 Demographic and clinical characteristics of the study population at baseline

\begin{tabular}{lcl}
\hline Total & $(N=108)$ & \\
Gender, $n(\%)$ & $61(57)$ & \\
Male & $47(43)$ & \\
Female & $65.3(11.7)$ & range: $38-93$ \\
Age at stroke, mean (SD) & $103.9(67.6)$ & range: $35-418$ \\
Time since stroke, days, mean (SD) & $99(92)$ & \\
Right handedness, $n(\%)$ & & \\
Type of stroke, $n(\%)$ & $15(14)$ & \\
Haemorrhage & $85(79)$ & \\
Infarction & $8(7)$ & \\
Otherwise/unknown & $20(19)$ & \\
Recurrent stroke, $n(\%)$ & $97(90)$ & \\
Hemiplegia/hemiparesis, $n(\%)$ & $22(20)$ & \\
Urinary incontinence, $n(\%)$ & $98(92)$ & \\
Sitting balance, $n(\%)$ & $54(50)$ & \\
Treatment, $n(\%)$ & $54(50)$ & range: $8-87$ \\
Strategy training & & range: $0-100$ \\
Usual treatment & $73(68)$ & range: $1-20$ \\
Type of institution, $n(\%)$ & $35(32)$ & range: $1.25-3.00$ \\
$\quad$ Rehabilitation centre & $59.7(19.7)$ & \\
Nursing home & $45.8(31.0)$ & \\
Apraxia test, mean (SD) & $11.0(4.9)$ & \\
Motricity Index, mean (SD) & $2.3(0.5)$ & \\
Barthel ADL Index, mean (SD) & & \\
ADL-observations, mean (SD) &
\end{tabular}


Donkervoort M, Dekker J, Deelman B.

The course of apraxia and ADL functioning in left hemisphere stroke patients treated in rehabilitation centres and nursing homes.

Clinical Rehabilitation: 20, 2006, nr. 12, p. 1085-1893

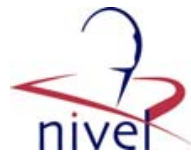

Table 2 Correlations between baseline scores and changes in apraxia and ADL functioning

\begin{tabular}{|c|c|c|c|c|c|c|}
\hline \multirow[b]{2}{*}{ Time period (weeks) } & \multicolumn{2}{|c|}{ Change on apraxia test } & \multicolumn{2}{|c|}{ Change on ADL-observations } & \multicolumn{2}{|c|}{ Change on Barthel ADL Index } \\
\hline & $0-8$ & $0-20$ & $0-8$ & $0-20$ & $0-8$ & $0-20$ \\
\hline Gender, male $(0 / 1)$ & 0.11 & $0.22^{*}$ & 0.09 & 0.13 & -0.02 & 0.13 \\
\hline Age at stroke & -0.01 & -0.15 & -0.05 & -0.03 & -0.08 & $-0.24^{*}$ \\
\hline Time since stroke & $-0.28^{* *}$ & -0.17 & -0.09 & -0.07 & $-0.33^{* *}$ & $-0.24^{*}$ \\
\hline Right handedness $(0 / 1)$ & 0.01 & -0.01 & -0.16 & -0.05 & 0.07 & 0.06 \\
\hline Type of stroke, infarction (0/1) & 0.05 & -0.06 & 0.03 & -0.05 & 0.12 & 0.14 \\
\hline Recurrent stroke $(0 / 1)$ & 0.07 & -0.02 & -0.02 & -0.04 & -0.14 & -0.11 \\
\hline Hemiplegia/hemiparesis $(0 / 1)$ & 0.07 & 0.15 & 0.11 & 0.11 & 0.04 & 0.20 \\
\hline Urinary continence $(0 / 1)$ & 0.06 & -0.08 & -0.13 & $-0.33^{* *}$ & -0.15 & $-0.23^{*}$ \\
\hline Sitting balance $(0 / 1)$ & 0.01 & -0.01 & 0.05 & -0.04 & -0.07 & -0.06 \\
\hline Type of institution, rehab centre $(0 / 1)$ & 0.20 & 0.18 & 0.15 & -0.05 & $0.22^{*}$ & $0.23^{*}$ \\
\hline Strategy training $(0 / 1)$ & 0.14 & 0.13 & 0.18 & -0.01 & $0.23^{*}$ & 0.03 \\
\hline Apraxia test & $-0.36 * *$ & $-0.46^{* *}$ & 0.04 & -0.17 & 0.01 & -0.04 \\
\hline Motricity Index & -0.01 & -0.17 & -0.03 & -0.05 & 0.09 & -0.15 \\
\hline Barthel ADL Index & - & - & -0.12 & -0.12 & $-0.24^{*}$ & $-0.36 * *$ \\
\hline ADL-observations & - & - & $-0.37 * *$ & $-0.52 * *$ & -0.01 & -0.08 \\
\hline
\end{tabular}

Table 3 Multiple regression models for prediction of change in apraxia and ADL functioning

\begin{tabular}{|c|c|c|c|c|c|c|}
\hline \multirow{2}{*}{$\begin{array}{l}\text { Time period: } \\
\text { Dependent variable }\end{array}$} & \multicolumn{3}{|l|}{ Week 0-8 } & \multicolumn{3}{|l|}{ Week 0-20 } \\
\hline & $\begin{array}{l}\text { Regression } \\
\text { model }\end{array}$ & $\begin{array}{l}\text { Regression } \\
\text { coefficient } \\
\text { beta }\end{array}$ & $\begin{array}{l}\text { Change in } \\
R^{2}\end{array}$ & $\begin{array}{l}\text { Regression } \\
\text { model }\end{array}$ & $\begin{array}{l}\text { Regression } \\
\text { coefficient } \\
\text { beta }\end{array}$ & $\begin{array}{l}\text { Change in } \\
R^{2}\end{array}$ \\
\hline Change on apraxia test & $\begin{array}{l}\text { Treatment }{ }^{\mathrm{a}} \\
\text { Apraxia }(0)^{\mathrm{b}} \\
\text { Time since } \\
\text { stroke } \\
\text { Total } R^{2}\end{array}$ & $\begin{array}{l}0.09 \\
-0.36^{* *} \\
-0.27^{* *}\end{array}$ & $\begin{array}{l}0.02 \\
0.13^{* *} \\
0.07^{* *} \\
0.22^{* *}\end{array}$ & $\begin{array}{l}\text { Treatment }^{\mathrm{a}} \\
\text { Apraxia }(0)^{\mathrm{b}} \\
\text { Gender } \\
\text { Total } R^{2}\end{array}$ & $\begin{array}{l}0.09 \\
-0.45^{* *} \\
0.24^{*}\end{array}$ & $\begin{array}{l}0.02 \\
0.20^{* *} \\
0.05^{* *} \\
0.27 * *\end{array}$ \\
\hline $\begin{array}{c}\text { Change on ADL- } \\
\text { observations }\end{array}$ & $\begin{array}{l}\text { Treatment }^{\mathrm{a}} \\
\text { ADL-obs }(0)^{\mathrm{b}} \\
\text { Apraxia } \\
\text { Age } \\
\text { Total } R^{2}\end{array}$ & $\begin{array}{c}0.23^{*} \\
-0.52^{* *} \\
0.26^{*} \\
-0.23^{*}\end{array}$ & $\begin{array}{l}0.03 \\
0.13^{* *} \\
0.05^{* *} \\
0.04^{* *} \\
0.25^{* *}\end{array}$ & $\begin{array}{l}\text { Treatment }{ }^{\mathrm{a}} \\
\text { ADL-obs }(0)^{\mathrm{b}} \\
\text { Barthel }(0)^{\mathrm{b}} \\
\text { Continence } \\
\text { Age } \\
\text { Total } R^{2}\end{array}$ & $\begin{array}{l}-0.03 \\
-0.66^{* *} \\
0.42^{* *} \\
-0.34^{* *} \\
-0.23^{*}\end{array}$ & $\begin{array}{l}0.00 \\
0.27^{* *} \\
0.04^{* *} \\
0.06^{* *} \\
0.04^{* *} \\
0.41^{* *}\end{array}$ \\
\hline $\begin{array}{l}\text { Change on Barthel ADL } \\
\text { Index }\end{array}$ & $\begin{array}{l}\text { Treatment }{ }^{\mathrm{a}} \\
\text { Barthel }(0)^{\mathrm{b}} \\
\text { Time since } \\
\text { stroke } \\
\text { Motricity Index } \\
\text { Age } \\
\text { Total } R^{2}\end{array}$ & $\begin{array}{c}0.20^{*} \\
-0.58^{* *} \\
-0.40^{* *} \\
0.32^{* *} \\
-0.23^{*}\end{array}$ & $\begin{array}{l}0.06^{*} \\
0.13^{* *} \\
0.10^{* *} \\
0.04^{* *} \\
0.03^{* *} \\
0.36^{*}\end{array}$ & $\begin{array}{l}\text { Treatment } \\
\text { Barthel }(0)^{\mathrm{b}} \\
\text { Time since } \\
\text { stroke } \\
\text { Age } \\
\text { Total } R^{2}\end{array}$ & $\begin{array}{c}0.05 \\
-0.54^{* *} \\
-0.35^{* *} \\
-0.28^{* *}\end{array}$ & $\begin{array}{l}0.00 \\
0.13^{* *} \\
0.15^{* *} \\
0.07^{* *} \\
0.35^{* *}\end{array}$ \\
\hline
\end{tabular}

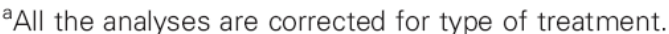

${ }^{\mathrm{b}}$ At baseline.

${ }^{*} P \leq 0.05 ; * * P \leq 0.01$. 
Donkervoort M, Dekker J, Deelman B.

The course of apraxia and ADL functioning in left hemisphere stroke patients treated in rehabilitation centres and nursing homes.

Clinical Rehabilitation: 20, 2006, nr. 12, p. 1085-1893
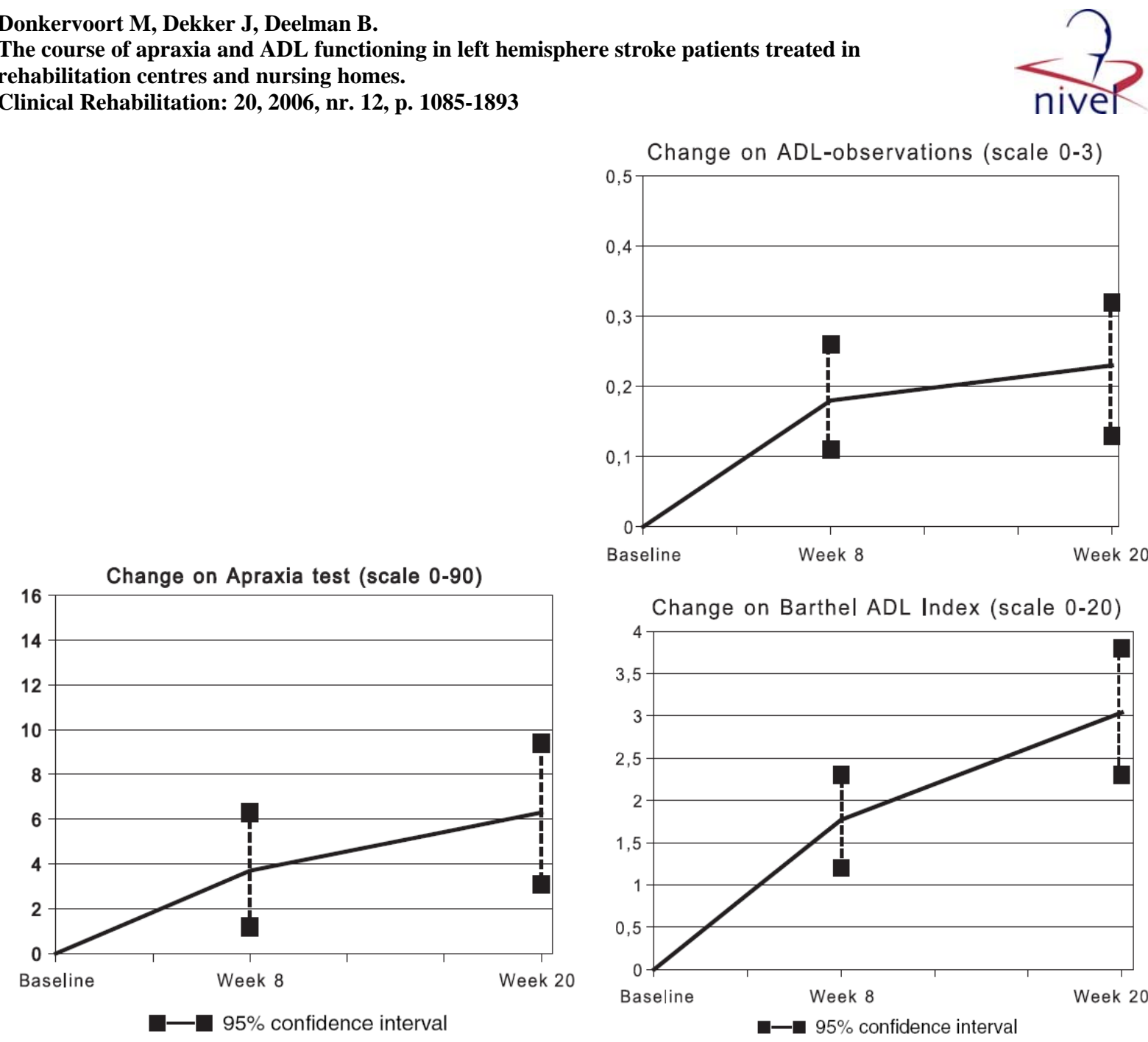

Change on ADL-observations (scale 0-3)

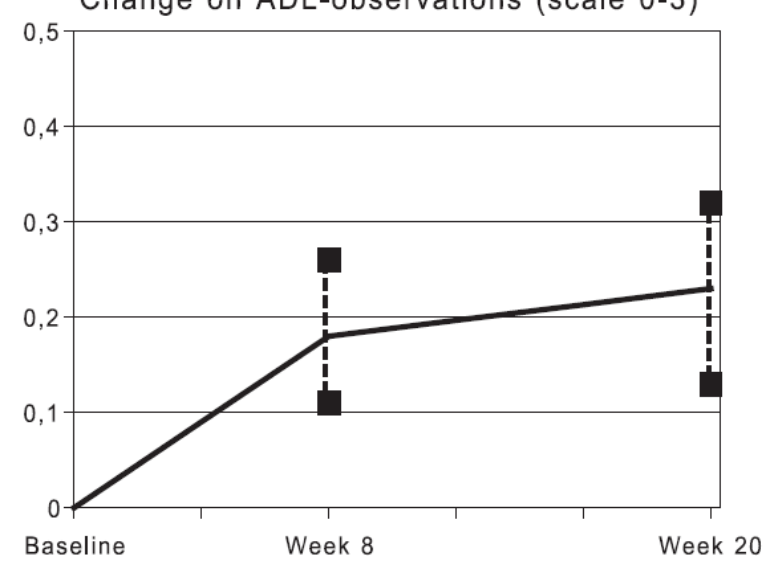

Change on Barthel ADL Index (scale 0-20)

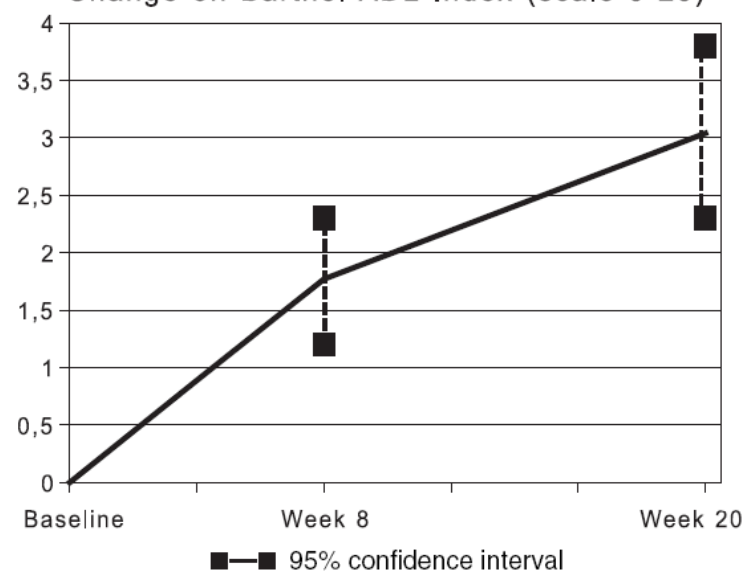

Figure 1 Course of apraxia.

Figure 2 Course of ADL functioning.

\section{REFERENCES}

1. De Renzi E. Apraxia. In Boller F, Grafman J eds. Handbook of neuropsychology, Vol. 2. Elsevier Science Publisher, 1989.

2. Kolb B, Wishaw IQ. Fundamentals of human neuropsychology, third edition. Freeman, 1990.

3. Rothi LJG, Heilman KM. Apraxia: The neuropsychology of action. Psychology Press, 1997.

4. Donkervoort M, Dekker J, Ende E van den, Stehmann-Saris JC, Deelman BG. Prevalence of apraxia among patients with a first left hemisphere stroke in rehabilitation centres and nursing homes. Clin Rehabil 2000; 14: 130-36.

5. Zwinkels A, Geusgens C, Sande P van de, Heugten C van. Assessment of apraxia: inter-rater reliability of a new apraxia test, association between apraxia and other cognitive deficits and prevalence of apraxia in a rehabilitation setting. Clin Rehabil 2004; 18: 819-27.

6. Poeck K. Clues to the nature of disruptions to limb praxis. In Roy EA ed. Neuropsychological studies of apraxia and related disorders. North-Holland, 1985.

7. Basso A, Capitani E, Della Sala S, Laiacona M, Spinnler, H. Ideomotor apraxia: a study of initial severity. Acta Neurol Scand. 1987; 76: 142-46.

8. Maher LM, Ochipa C. Management and treatment of limb apraxia. In Rothi LJG, Heilman KM eds. Apraxia: The neuropsychology of action. Psychology Press, 1997.

9. Foundas AL, Raymer AM, Maher LM, Rothi LJG, Heilman KM. Recovery in ideomotor apraxia. J Clin Exp Neuropsychol 1993; 15: 44.

10. Bjorneby El, Reinvang IR. Acquiring and maintaining self-care skills after stroke. Scand J Rehabil Med 1985; 17: 75-80.

11. Sundet K, Finset A, Reinvang I. Neuropsychological predictors in stroke rehabilitation. J Clin Exp Neuropsychol 1988; 10: 363-79.

12. Jong MC de, SCC Hartong, GJ Lankhorst. Functional outcome of stroke patients: an exploratory study in an outpatient rehabilitation department. J Rehabil Sci 1992; 5: 14-21. 
Donkervoort M, Dekker J, Deelman B.

The course of apraxia and ADL functioning in left hemisphere stroke patients treated in rehabilitation centres and nursing homes.

Clinical Rehabilitation: 20, 2006, nr. 12, p. 1085-1893

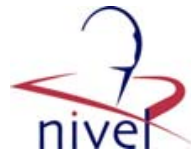

13. Foundas AL, Macauley BC, Raymer AM, Maher LM, Heilman KM, Rothi LJG. Ecological implications of limb apraxia: evidence from mealtime behavior. J Int Neuropsychol Soc 1995; 1: 62-66.

14. Giaquinto S, Buzelli S, Di Francesco L, Lottarini A, Montenero P, Tonin P, Nolfe G. On the prognosis of outcome after stroke. Acta Neurol Scand 1999; 100: 202-208.

15. Hanna-Pladdy B, Heilman KM, Foundas AL. Ecological implications of ideomotor apraxia: evidence from physical activities of daily living. Neurology 2003; 60: 487-90.

16. Donkervoort M, Dekker J, Stehmann-Saris JC, Deelman BG. Efficacy of strategy training in left hemisphere stroke patients with apraxia: a randomized clinical trial. Neuropsychol Rehabil 2001; 11: 549-66.

17. World Health Organization. Special Report: Recommendations on stroke prevention, diagnosis and therapy. Stroke 1989; 20: 1407-31.

18. Heugten CM van, Dekker J, Deelman BG, Stehmann-Saris JC, Kinebanian A. A diagnostic test for apraxia in stroke patients: internal consistency and diagnostic value. Clin Neuropsychol 1999; 13: 1-11.

19. Demeurisse G, Demol O, Robaye E. Motor evaluation in vascular hemiplegia. Eur Neurol 1980; 19: 382-89.

20. Collen FM, Wade DT, Bradshaw CM. Mobility after stroke: reliability of measures of impairment and disability. Int Disabil Stud 1990; 12: 6-9.

21. Wade DT. Measurement in neurological rehabilitation. Oxford University Press, 1992.

22. Collin C. Wade D. Assessing motor impairments after stroke: a pilot reliability study. J Neurol Neurosurg Psychiatry 1990; 53: 576-79.

23. Heugten CM van, Dekker J, Deelman BG, Stehmann-Saris JC, Kinebanian A. Assessment of disabilities in stroke patients with apraxia: internal consistency and inter-observer reliability. Occup Ther J Res 1999; 19: 55-73.

24. Heugten CM van, Dekker J, Deelman BG, Dijk AJ van, Stehmann-Saris JC, Kinebanian A. Measuring disabilities in stroke patients with apraxia: a validation study of an observational method. Neuropsychol Rehabil 2000; 10: 401-14.

25. Collin C, Wade DT, Davies S, Horne V. The Barthel ADL Index: a reliability study. Int Disabil Stud 1988; 10: 61-63.

26. Wade DT, Collin C. The Barthel ADL Index: a standard measure of physical disability? Int Disabil Stud 1988; 10: 64-67.

27. Cohen J. Statistical power analysis for the behavioral sciences. Lawrence Erlbaum Associates, 1988.

28. Wade DT, Hewer RL. Functional abilities after stroke: Measurement, natural history and prognosis. J Neurol Neurosurg Psychiatry 1987; 50: 177-82.

29. Wolfe CDA, Taub NA, Woodrow EJ, Burney PGJ. Assessment of scales of disability and handicap for stroke patients. Stroke 1991; 22: 1242-44.

30. Donkervoort M, Dekker J, Deelman BG. Sensitivity of different ADL measures to apraxia and motor impairments. Clin Rehabil 2002; 16: 299-305.

31. Kwakkel G, Wagenaar RC, Kollen B, Lankhorst GJ. Predicting disability in stroke: a critical review of the literature. Age Ageing 1996; 25: 479-89. 